\title{
Satellite Cognitive Communications: Interference Modeling and Techniques Selection
}

\author{
Shree Krishna Sharma, Symeon Chatzinotas, and Björn Ottersten \\ SnT - securityandtrust.lu, University of Luxembourg \\ Email:\{shree.sharma, symeon.chatzinotas, bjorn.ottersten\}@uni.lu
}

\begin{abstract}
Due to increasing demand of high speed data rate for satellite multimedia and broadcasting services and spectrum scarcity problem in satellite bands, exploring new techniques for enhancing spectral efficiency in satellite communication has become an important research challenge. In this aspect, satellite cognitive communication can be considered as a promising solution to solve spectrum scarcity problem. In this paper, different cognitive techniques such as underlay, overlay, interweave and database related techniques are discussed by reviewing the current state of art. Exact beam patterns of a multi-beam satellite are plotted over the Europe map and interference modeling between terrestrial Base Station (BS) and satellite terminal is carried out on the basis of interference power level. Furthermore, suitable cognitive techniques are proposed in high and low interference regions in the context of satellite cognitive communication.

Index Terms-Satellite Communication, Cognitive Radio, Interference, Spectral Sensing, Underlay, Overlay
\end{abstract}

\section{INTRODUCTION}

The increasing demand for high speed wireless internet as well as digitized audio and video is leading to a rapidly increasing market for wireless multimedia services. However, the available frequency resources are becoming scarce due to the spectrum segmentation and the dedicated frequency allocation of the standardized wireless systems. Therefore, it becomes an important research challenge to define and investigate new network architectures which have the ability to support higher system throughput and energy efficiency, providing large-scale coverage. Because of the increase in spectrum demand, current static allocation policy faces spectrum scarcity while a significant amount of spectrum remains underutilized for almost $90 \%$ of time [1]. In the context of satellite communication, due to high demand of broadband services and limitation of available $\mathrm{L}$ and S-band frequency resources, higher frequency bands i.e. $\mathrm{Ku}$ and $\mathrm{Ka}$ bands have been assigned for fixed satellite services [2]. There has been continued pressure on satellite bands, especially in L and C bands due to the introduction of new terrestrial services such as $3 \mathrm{G}$ mobile telephony, WiMax and WiFi services .

Different fundamental physical constraints such as power requirements, terrain and infrastructure obstructions and antenna types etc. for wide area coverage may cause the terrestrial wireless communication to be dependent on satellite communication [3]. The satellite technology is able to supplement the drawbacks experienced by terrestrial systems to extend the coverage. Due to this important aspect, the hybrid/integrated system has been considered as an important research field and has been investigated in much literature [3-6]. Heterogeneous network design trends grow faster as the demand for anytime, anywhere, and anyway service increases [5]. Satellite communication plays an important role in building such heterogeneous architectures through hybrid and integrated satellite/terrestrial paradigms.

Static allocation of the frequency spectrum in traditional ways does not meet the requirements of future wireless networks. Therefore, dynamic spectrum sensing, allocation and management is required for the co-existence of different terrestrial and satellite networks on the same spectrum band. Due to the limited and expensive bandwidth, Cognitive Radio (CR) can be an efficient way to increase spectrum efficiency for satellite network [7]. This technique has the strong potential to unlock spectral gridlock with the help of advanced radio design techniques, powerful encoding and signal processing techniques [8]. In satellite cognitive scenario, suitable cognitive techniques can be designed to help satellite users, which are in low Signal to Noise Ratio (SNR) condition, and to provide service to terrestrial users while preserving the Quality of Service (QoS) of the satellite link by optimal utilization of the spectrum. The following conditions, which are unique to Satellite Communications (SatComs), have differentiated the cognitive SatCom research area from the terrestrial one.

- Dynamic spectrum sensing becomes difficult to be carried out in satellite downlink due to large coverage area and weak signal level, even in case of multispot.

- The resource management problem becomes difficult in the co-existence scenario of satellite and terrestrial networks or two different satellite networks.

- Uplink transmissions with low elevation angles tend to interfere more with terrestrial systems compared to the uplinks with higher elevation angle.

- Satellite receivers in high latitude suffer from more interference coming from terrestrial BSs than the receivers near the equator region.

- Polarization and elevation angle can be considered as two different separate degrees of freedom while analyzing interaction with terrestrial systems.

There are various research works in literature related to terrestrial CR but still much research work has not been performed in the context of cognitive SatComs. Satellite and terrestrial networks can be considered as primary and secondary systems respectively or viceversa. In many scenarios, 
the satellite transmission should be given high priority mainly due to its adverse transmission conditions and poor indoor coverage. However, in some scenarios, point to point terrestrial microwave links can be used as primary link and satellite link can be used as secondary by sharing the same microwave frequency band. In the direction of interference modeling, there are much recent works in the context of terrestrial CR networks [9-12]. According to authors' knowledge, no research has been reported in the literature considering the selection of cognitive techniques based on interference power level in the context of cognitive SatComs. This is the main focus of this paper. A satellite terrestrial cognitive scenario has been considered and interference modeling between a fixed satellite (SAT) terminal and a terrestrial BS is carried out to select the best cognitive technique. Interference between a fixed satellite terminal and a terrestrial BS depends on geographical location as well as distance between them. Depending on the high or low interference level, suitable cognitive techniques are proposed.

The remainder of this paper is structured as follows: Section II presents the overview of literature related to different cognitive techniques. Section III presents interference modeling and provides motivation for carrying out research in this field. Section IV presents the cognitive technique selection criteria and applicable techniques. Section V concludes this paper by providing overall conclusion.

\section{RELATED WORK}

In the field of satellite communications, the main available literature related to cognitive techniques has considered hybrid or integrated satellite networks. In hybrid satellite-terrestrial network, the network management of the satellite and the terrestrial component is performed separately with the collaboration of a satellite and a terrestrial operator, whereas, in an integrated systems, the network management is carried out by a single entity [4]. In hybrid or integrated scenario, a satellite system based on DVB-SH has been taken as a primary system and terrestrial wireless network as a secondary system in [13]. A number of research works has been performed mainly focusing on the coexistence of a satellite and terrestrial communication system over the same bandwidth in [4-6, 13]. The concept of cognitive satellite terrestrial radios for hybrid satellite-terrestrial systems has been presented in [3]. There have been some research works in literature in the direction of distributed power allocation for CR systems. In [14], a distributed power allocation scheme has been proposed for $\mathrm{CR}$ and in [15], a power allocation scheme for CR networks based on game theory has been proposed. Furthermore, power allocation strategies for CR terminals in the context of satellite communication have been proposed in [16]. Most of research works in the literature have studied underlay or overlay strategies dealing with the constraints on interference from the secondary system to the primary system by employing power control or Ultra Wide-band spreading methods. Recently, due to rapid switchover of analog $\mathrm{TV}$ to digital $\mathrm{TV}$, a portion of $\mathrm{TV}$ analog channels become entirely vacant due to the higher spectrum efficiency of digital TV [17]. According to modeling studies commissioned by Ofcom, over $50 \%$ of locations in the UK are likely to have more than $150 \mathrm{MHZ}$ of interleaved spectrum and that even at $90 \%$ of locations around $100 \mathrm{MHz}$ of interleaved spectrum might be available for cognitive access [18]. In [19], it is suggested that there is significant scope for license-exempt use of the released defense spectrum using CR technology, both on a spatial and a temporal basis.

Cognitive techniques can be divided into centralized and distributed based on the network architecture and can be classified into cooperative or non-cooperative according to the access behavior of Secondary Users (SUs). The cognitive techniques considered in literature can be basically categorized into 4 types on the basis of access technology of SUs. Main features of these four techniques are summarized in table I and some research works related to these techniques are presented in the following subsections.

\section{A. Interweave or Spectrum Sensing Technique}

This technique, also called interference avoidance technique, requires SUs to communicate using spectral holes in space, time, and frequency, which are not occupied by Primary Users (PUs). Therefore, no interference occurs in the ideal case [8]. In [20], two switch model and opportunistic channel selection methods are proposed for implementing this interweave technique. In [21], spectrum shaping has been proposed as a technique to improve spectrum efficiency in CR networks and interference fitting as well as licensed signal avoidance techniques have been considered. There are several Spectrum Sensing (SS) techniques proposed in literature for CR based systems. The three mainly used signal processing techniques for sensing in literature are Matched filter detection, Energy Detection (ED) and Cyclostationary feature detection [8]. The performance of these three techniques is limited by received signal strength which may be severely degraded in multi-path fading and shadowing environment. In such cases, cooperative sensing can be considered to facilitate the problem of detecting the PU by reducing the probability of interference to a PU [22]. In this method, a large network of cognitive radios with sensing information exchanged between neighbors gets a better chance of detecting the PU compared to individual sensing. In addition, cooperation can solve hidden primary user problem and it can decrease the sensing time [22]. The polarization domain and the directivity of antenna patterns can be exploited as extra degree of freedoms for implementing cognitive technique. In [23], the polarization domain has been exploited in the context of cognitive SatComs in a dual polarized AWGN channel. In [24], ED based SS technique has been analyzed in a dual polarized Rayleigh fading channel in the context of cognitive SatComs.

\section{B. Underlay Technique}

Underlay networks are characterized by strict constraints on the interference that may be caused by a SU to PUs. The co-existence of satellite and terrestrial systems is possible in 
TABLE I: Features of different cognitive techniques

\begin{tabular}{|c|c|c|c|c|}
\hline Cognitive Techniques & Spectrum Sensing (SS) & Underlay (UL) & Overlay (OL) & Database (DB) \\
\hline Cognitive Information & Power Spectral Density (PSD) & $\begin{array}{l}\text { Channel State } \\
\text { Information (CSI) }\end{array}$ & CSI and data & Geolocation data \\
\hline QoS Constraints & $\begin{array}{l}\text { Probability of Detection } \\
\text { Probability of False alarm }\end{array}$ & $\begin{array}{l}\text { Interference Threshold, } \\
\text { Interference Temperature }\end{array}$ & Rate limit threshold & Access Time \\
\hline Problems to be solved & $\begin{array}{l}\text { Wide area sensing, } \\
\text { Weak signal detection }\end{array}$ & $\begin{array}{l}\text { Resource optimization, } \\
\text { Interference Control }\end{array}$ & $\begin{array}{l}\text { Interference Mitigation, } \\
\text { Requirement of efficient } \\
\text { coding technique }\end{array}$ & $\begin{array}{l}\text { Database } \\
\text { Management } \\
\text { Problem }\end{array}$ \\
\hline Applicable Techniques & $\begin{array}{c}\text { Energy Detection, } \\
\text { Matched Filter Detection, } \\
\text { Cyclostationary Feature Detection, } \\
\text { Spectral Shaping, } \\
\text { Polarization sensing, } \\
\text { Collaborative Sensing, } \\
\text { Compressive Sensing, }\end{array}$ & $\begin{array}{l}\text { Power and Radio } \\
\text { resource allocation, } \\
\text { Beamforming and } \\
\text { Scheduling Techniques } \\
\\
\text { Multi-antenna and } \\
\text { diversity techniques }\end{array}$ & $\begin{array}{l}\text { Superposition Coding, } \\
\text { Rate splitting, } \\
\text { Relaying, } \\
\text { Known Interference } \\
\text { precancelation }\end{array}$ & $\begin{array}{c}\text { Efficient } \\
\text { Database } \\
\text { access Techniques }\end{array}$ \\
\hline
\end{tabular}

underlay scheme, only if the interference caused by the terrestrial transmitters to the satellites system can be controlled and managed properly [25]. In underlay technique, the maximum allowable interference level at the PU's Receiver $(\mathrm{Rx})$ can be modeled by the concept of interference temperature, as defined by the FCC Spectrum Policy Task Force in [26]. This metric guarantees the reliable operation of the PUs regardless of SU's spectrum utilization [27]. The unlicensed transmitters utilizing this band must guarantee that their transmission added to the existing interference must not exceed that limit at a licensed Rx. The concept of interference alignment (IA) has been illustrated in [28]. IA refers to the technique that designs signals so that they form shadows at the receivers when they constitute interference and remain distinguishable where they are desired. Considering an underlay spectrum sharing scheme, researchers have also contributed in the robust beamforming design with regard to imperfect CSI available at secondary transmitters in order to maximize some performance metrics in the secondary network [29,30]. Diversity techniques and adaptive modulation techniques have been proposed in literature to meet the interference constraint in underlay $\mathrm{CR}$ networks. The concept of adaptive modulation is to adapt the modulation parameters, such as constellation size, to fading channel conditions by maintaining the desired QoS and it can achieve higher spectral efficiency over wireless channels than traditional modulation schemes.

\section{Overlay Technique}

Overlay networks are characterized by the mitigation of interference with the help of advanced coding strategies at the cognitive transmitters that facilitate communications for other users. In this technique, the cognitive transmitter should have knowledge of the PU's codebooks and possibly its messages as well [8]. By using sophisticated coding techniques, this information can be used to completely cancel the interference at cognitive receiver due to PUs and to assign a part of their power for their own communication and remaining power to assist in primary transmissions. Different Pre-coding techniques such as Gel'fand-Pinsker binning and dirty paper coding in Gaussian channels can be applied to overlay networks [31] [32]. In [33], an overlay SU signal over a satellite communication channel occupied by a PU has been considered, the SU is allowed to transmit data symbols as a fraction of the PU's symbol rate. In [34], a linear search based approach is proposed to optimally allocate the transmit power and design the beamformers in an overlay CR channel with a single primary and a single cognitive user, where the cognitive user causally obtains primary message knowledge at its transmitter. Overlay techniques are the most suitable techniques for integrated systems where there is a high degree of cooperation between satellite and terrestrial networks.

\section{Database technique}

Dynamic spectrum management can be performed through databases. Radio environment maps (REM) are becoming a promising architectural concept for storing environmental information for use in cognitive wireless networks. In [35], it has been shown that the enhancement of the REM by means of spatial statistics and models can be obtained through a topology engine, which is an agent collecting and processing spatial information about the environment for storage in the REM. One of the sensing and measuring schemes is the wireless regional area network (WRAN) system based on REM as proposed in [36], which can be looked as an integrated database. REM enabled WRAN system has been proposed in [37] and it has been shown that it can better realize different functions at the same time to ensure the protection of PUs. In case of cognitive access to TV white space scenario, based on CR's geolocation data and specific service requirements, the home base station queries the central database for channel availability [38]. The database then returns information about various operating parameters such as number of channels, 
centre frequency and associated power levels available for use in that location.

\section{INTERFERENCE MODELING}

The proposed satellite terrestrial cognitive scenario comprises of two communication links sharing the spectrum. These links can be i) from satellite to fixed SAT terminal, ii) from terrestrial BS to terrestrial user terminal, iii) from fixed SAT terminal to satellite, iv) from terrestrial user terminal to terrestrial BS. In this context, two priority conditions can be set i.e. by providing primary access to satellite or to terrestrial link. In many cases, satellite link can be considered as primary link due to adverse transmission conditions and in some of the cases, terrestrial link can be considered as primary. The latter situation can be beneficial for satellite operators to take advantage of terrestrial spectrum. An important application for satellite cognitive scenario can be a satellite network operating in $\mathrm{C}$ band and terrestrial WiMax network. Although there has been long debate on the interference issue of WiMax service to satellite downlink transmission on current deployed scenario, current practical cases do not use cognitive techniques to share the frequency band. These two systems can be made to operate in the same spectrum by using suitable cognitive techniques. Another scenario for this network architecture would be the exploitation of the VHF analog spectrum which is going to be available after the switch to DVB-T. This spectrum could be shared by a satellite to vehicle service and a terrestrial mobile network. Underlay technique can be used between two systems by ensuring that the interference level does not exceed the interference threshold required by PUs. Suitable overlay cognitive techniques can be exploited in order to help satellite users who are in low SNR condition, and to provide some service to terrestrial users without degrading the QoS of the satellite link. Although these two tasks seem to be contradictory, the terrestrial BS can exploit the cognition of the satellite messages and achieve an optimal utilization of the available spectrum. SS or interweave techniques can identify the vacant spectrum and allocate those vacate bands for secondary access.

Each of the cognitive techniques has different requirements and different levels of applicability to the considered scenario. Interference modeling can be used as a tool to select the best technique among them depending on the cognitive scenario and geographic region. The cognitive technique suitable in one geographic location and for a certain condition may not be suitable for other locations and conditions. It is quite important to select suitable cognitive technique for efficient operation of $\mathrm{CR}$ networks in the context of co-existence of satellite terrestrial networks. In the following subsections, we present different transmission modes for satellite terrestrial cognitive scenario and model the interference based on interference power level to select the best cognitive technique depending on the geographical location.

\section{A. Transmission Modes}

Considering the co-existence of satellite (SAT) and terrestrial network in the same spectrum, there can be 4 different

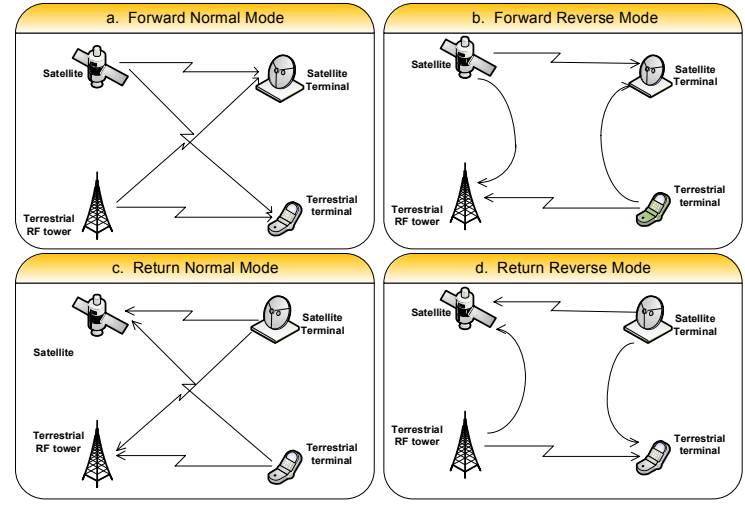

Fig. 1: Different transmission modes in satellite cognitive scenario

modes of operation as shown in Fig. 1. They are : a) Forward Normal mode, b) Forward Reverse Mode, c) Return Normal Mode, and d) Return Reverse Mode. Out of these transmission modes, only forward normal mode and return normal modes have been considered in literature for the analysis of integrated systems. The interference from satellite to terrestrial terminal/terrestrial tower as well as from terrestrial terminal/ tower to the satellite can be considered to be negligible due to large distance in all of these scenarios. Although practical transmission mode depends on current ITU-R regulation and the mode selection is not under the control of researchers, we are interested at all possible combinations for our research work. In this paper, we focus on interference power levels to classify weak or strong interaction between primary and secondary links. Furthermore, exploring the possibility of using cognitive techniques in all four types of modes for implementing effective cognitive satellite communication is an important aspect to be explored. Due to wide area coverage of satellite, it seems quite difficult to operate satellite forward link in secondary basis. However, there is a good opportunity to use satellite return link in secondary basis due to directive nature of transmission from satellite terminals.

\section{B. Interference Power Level}

For a geostationary satellite, multi-beam antennas can be equipped to cover multiple spots over the surface of the Earth. Multiple beams can be employed instead of a single global beam in order to cover the same coverage area to increase the capacity as in the case of terrestrial cellular paradigm. Nowadays, tens or hundreds of beams are possible with a frequency reuse factor of three or four [39]. In this work, we consider the coverage for whole European region with 135 beams. The exact 3-dB footprint coverage of SES satellite ASTRA 2D, a Geo-stationary satellite located at $28.2^{\circ} \mathrm{E}$, by considering multi-beam satellite has been plotted over the Europe map and has been presented in Fig. 2. The link budget and simulation parameters has been presented in table II. Beam contours are plotted by considering a multi-beam satellite using 135 beams, each of radius $165 \mathrm{Km}$. It can be observed that the $3-\mathrm{dB}$ footprint contours become more elongated as we move from equator to the polar region. To plot the beam 


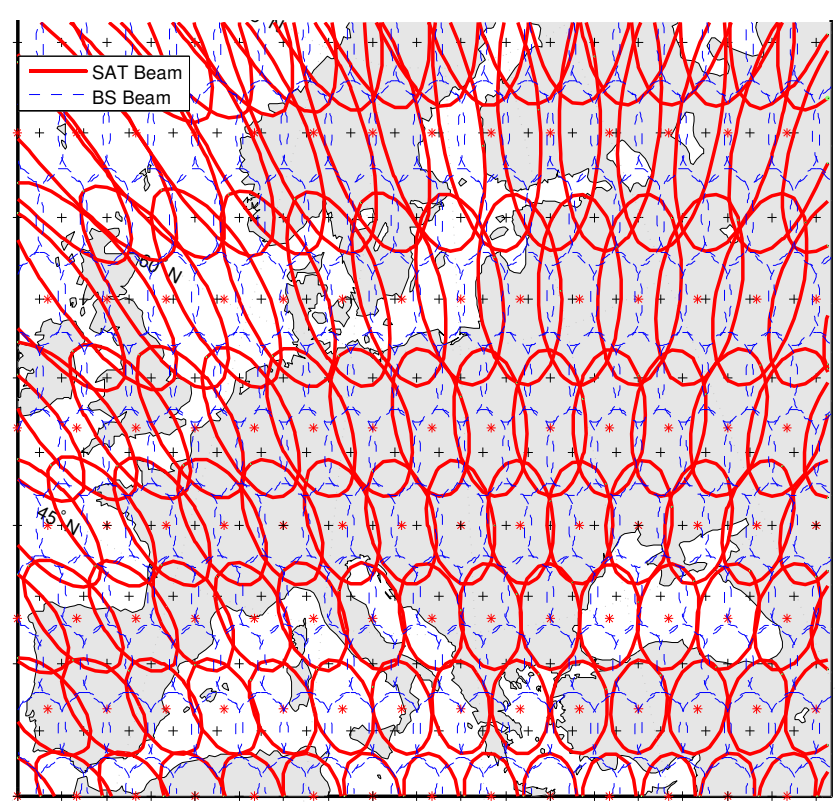

Fig. 2: Coverage of Europe with multispot beams

patterns, beam gain matrix has been used. Beam gain matrix of $k$-th beam for $m$-th user position can be denoted by $B(m, k)$ and can be written as [40]:

$$
B(m, k)=G_{\max } \cdot\left(\frac{J_{1}(u(m, k))}{2 u(m, k)}+36 \frac{J_{3}(u(m, k))^{2}}{u(m, k)^{3}}\right)^{2}
$$

where $u(m, k)=2.01723 \sin (\theta(m, k)) / \theta_{3 d B}, J_{i}$ is first kind of Bessel's function of order $i$, and $G_{\max }$ is the maximum antenna gain. $\theta_{3 d B}$ is the $3-\mathrm{dB}$ angle which is calculated by considering the minimum slant range for central beam and $\theta(m, k)$ represents the nadir angle to $m$-th user position from $k$-th beam center position with respect to satellite.

The slant ranges for the spots located in the polar region are longer than those in the equator region. By comparing path loss from satellite to different user points on the footprint of satellite, it can be observed that the path loss varies as we move away from the centre beam position, i.e. from the equator to the polar region in case of a geostationary satellite. The denser grids shown in the map are drawn while placing terrestrial BS and their footprint coverage areas have been drawn with circular approximation. It has been assumed that SAT terminal is equipped with an offset fed parabolic dish antenna. The radiation pattern of an offset fed parabolic antenna in azimuth plane has been taken into consideration for the interference analysis with the terrestrial BS. One SAT terminal and one terrestrial BS antenna separated with a certain distance at different latitude points have been considered in the analysis for modeling simple scenario. Terrestrial BS towers are considered to be uniformly distributed within a circle of radius ranging from $0.5 \mathrm{Km}$ to $10 \mathrm{Km}$. Figure 3 shows interference power versus latitude of geographical location and distance between SAT terminal and BS antenna in the form of a $3 \mathrm{D}$ diagram. While analyzing the elevation angle range of points on Europe satellite map coverage, it has been

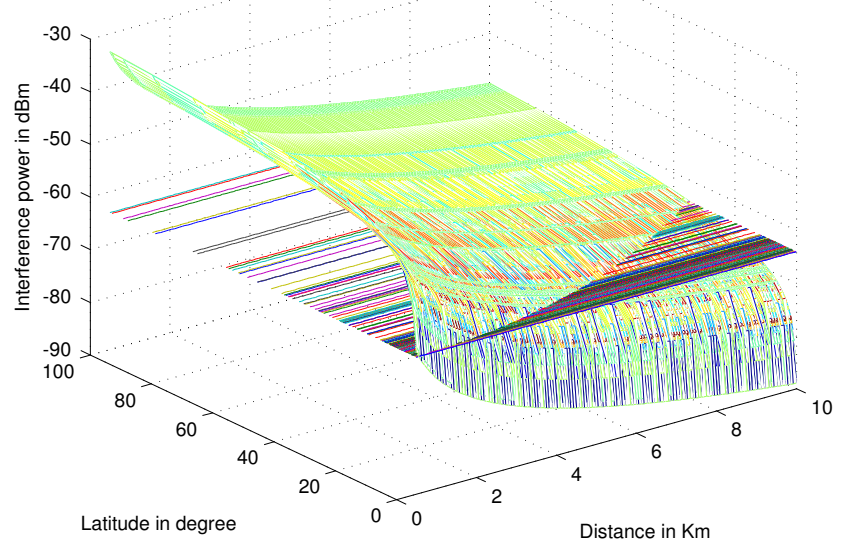

Fig. 3: Interference power versus latitude and distance

observed that it ranges from $7.07^{\circ}$ to $49.40^{\circ}$ for SES ASTRA 2D satellite. Due to this reason, SAT terminal antennas near the polar region are oriented to satellite with low elevation angles as compared with the antennas, which are located nearer to the equator. If we combine the effect of terrestrial BS interference with this phenomenon, it can be concluded that SAT terminal antennas suffer from more interference as we move to the north from the equator. From the result shown in Fig. 3, it can be deduced that the interference power increases as we move towards the polar region. For the interference modeling purpose, we consider $-64 \mathrm{dBm}$ as the tolerable interference threshold value i.e. interference below this value is tolerable to the SAT terminal. This threshold value has been chosen on the basis of dynamic frequency selection (DFS) threshold value for devices having EIRP values from $23 \mathrm{dBm}$ to $30 \mathrm{dBm}$ [41]. It should be noted that this threshold has been chosen as a reference for illustrating interference modeling method and may not be same in practical scenarios. From the simulation result presented in Fig. 3, it can be noted that interference mitigation techniques need to be considered for all the latitude range if distance between SAT terminal and terrestrial BS is less than $2 \mathrm{Km}$ and the interference becomes worse as we move towards north. If we consider the regions having elevation angles less than $30^{\circ}$ i.e. as we move from $52^{\circ}$ to the north for distances between SAT terminal and BS antenna up to $5 \mathrm{Km}$, the interference becomes worse. This region can be considered as high interference region and another region below $52^{\circ}$ can be regarded as tolerable interference region.

Furthermore, it can be noted that SAT terminal with elevation angle less than $30^{\circ}$ are in risk of getting more than $-64 \mathrm{dBm}$ interference from terrestrial BS for distances up to $5 \mathrm{Km}$. In terms of distances, in general, the interference needs to be accounted and handled carefully if the distances between SAT terminal and terrestrial BS is less than $5 \mathrm{Km}$. In case of SAT antennas oriented with elevation angle less than $10^{\circ}$, the separation distance at least $10 \mathrm{Km}$ needs to be taken into account. If we want to allow the co-existence of satellite terrestrial systems in the regions where the elevation angle 
TABLE II: Simulation \& Link Budget Parameters

\begin{tabular}{l|c} 
Parameter & Value \\
\hline Carrier frequency $f$ & $4 \mathrm{GHz}$ \\
Terrestrial BS RF power $P_{t}$ & $25 \mathrm{dBm}$ \\
Terrestrial BS antenna Gain $G_{t}$ & $10 \mathrm{~dB}$ \\
EIRP BS antenna & $35 \mathrm{dBm}$ \\
Distance bet SAT Ter and BS $d$ & $0.5 \mathrm{Km}$ to $10 \mathrm{Km}$ \\
Path loss range $L_{p} \propto d^{-2}$ & 98.47 to $124.49 \mathrm{~dB}$ \\
Satellite Terminal Gain range $G_{r}$ & 20 to $-9.5047 \mathrm{~dB}$ \\
Free Space Loss for SAT link & $196 \mathrm{~dB}$ \\
3-dB Beamwidth $\theta_{3 d B}$ & $0.253^{\circ}$ \\
Satellite terminal $G / T$ & $24 \mathrm{~dB} / \mathrm{K}$ \\
Beam Radius & $165 \mathrm{Km}$ \\
Satellite latitude & $28.2^{\circ} \mathrm{E}$ \\
Satellite height & $36000 \mathrm{Km}$ \\
Parabolic dish F/D ratio & $1 / 2$ \\
Considered latitude range & $35^{\circ}$ to $70^{\circ}$ \\
Considered Longitude range & $-10^{\circ}$ to $45^{\circ}$ \\
Elevation angle range & $7.07^{\circ}$ to $49.40^{\circ}$ \\
SAT terminal slant range & $37325 \mathrm{Km}^{\circ}$ to $41070 \mathrm{Km}$
\end{tabular}

is below $10^{\circ}$, interference mitigation techniques may not be sufficient to mitigate the interference and suitable SS and DB cognitive techniques need to be investigated.

\section{Techniques Selection}

\section{A. Selection Criteria}

As stated in section III, 4 categories of transmission modes are possible and general cognitive structure can be considered as $\mathrm{X}$ model as shown in Fig. 4. In each of these modes, the interference from satellite to terrestrial terminal/tower as well as from terrestrial terminal/ tower to the satellite can be considered to be negligible due to large distance. In this context, the $\mathrm{X}$ network model can be reduced to $\mathrm{Z}$ model. This reduction is possible in the co-existence of satelliteterrestrial networks and can be taken as an advantage over the co-existence scenario of two terrestrial networks. By using the interference modeling with regard to geographical location presented in this paper, the $\mathrm{X}$ model can be further reduced to a parallel model as shown in Fig. 4 for low interference regions. Parallel structure allows two networks to exist independently in the same spectrum. In low interference regions, there is possibility of co-existence of satellite and terrestrial networks in the same spectrum with very less interaction, whereas, in high interference regions, $\mathrm{Z}$ model need to considered by allowing sufficient interaction between satellite and terrestrial systems.

Two interference models can be considered: low interaction model for the regions below $52^{\circ}$ and distances more than 5 $\mathrm{Km}$ and high interaction model for the regions above $52^{\circ}$ and distances less than $5 \mathrm{Km}$. In each scenario, one link may be considered as strong and the other as weak, primary links always being strong. If the interference level is high, there arises less possibility of suppressing interference by using interference mitigation techniques. Complex mitigation and cognitive techniques need to be investigated in such context.
In this context, it is better to use SS and DB techniques for achieving best performance. Out of these two techniques, DB technique can be used from long-term perspective and SS technique from short-term perspective. If the interference level is low or medium, interference from one system to another system can be suppressed by using some form of underlay technique. Overlay technique can be used in both high interference and low interference scenario by using advanced coding and modulation techniques. Since sufficient level of interaction between primary and secondary system is required in overlay systems, this technique appears to be complicated from practical aspects in the considered scenario.

Furthermore, satellite terminal antennas have the special feature to always look to the equator in case of a Geostationary satellite. This feature can be used as an additional degree of freedom in analyzing the interference. In addition, it can be used for Direction of Arrival (DoA) aware beamforming based underlay techniques by using the priori knowledge of DoA of satellite signals. Moreover, proper design of radiation patterns can exploit another degree of freedom for the coexistence of satellite and terrestrial networks on the same spectrum. Depending on the latitude, satellite is seen under a high elevation angle and proper design of radiation patterns can allow the reduction of interference between primary and secondary systems. This fact facilitates 3D space-time-frequency utilization in satellite $\mathrm{CR}$ networks rather than 2D timefrequency utilization in terrestrial CR networks [3].

In general, satellite antennas use circular polarization and terrestrial BS antennas use linear polarization. The polarization domain can be considered as an additional degree of freedom to isolate the interference between primary and secondary systems. By deploying two orthogonally polarized antennas, any type of receive or transmit polarization can be derived. If the polarization types of the signal and receiving antenna are matched with each-other, then maximum power of arriving signal is transferred to the antenna. The polarization domain can be used form the perspective of sensing the PU signal as well as increasing the throughput of primary and secondary systems. The polarization domain has been exploited for SS purpose in a dual polarized AWGN channel in our previous work [23]. In another work [24], ED based SS technique has been analyzed for a dual polarized fading channel in the context of cognitive SatComs.

\section{B. Applicable Techniques}

Regarding SS technique, there are many techniques proposed in literature and research works are being carried out in dynamic SS techniques with different new domains such as elevation angle, polarization etc. Since SS in satellite cognitive scenario needs to be carried out in wide area, collaboration of terrestrial BSs to aid in satellite SS can be one important technique in the considered scenario. Exploring the applicability of collaborative SS can be an important future area. This technique can also be exploited to solve the Hidden Node Problem (HNP) of satellite communication. Among many available SS techniques, ED is the most common because of 
its low computational and implementation complexities [42]. The signal is detected by comparing the output of the energy detector with a threshold which depends on the noise floor [43]. The optimal way for any signal detection is a matched filter since it maximizes received SNR. The main advantage of matched filter is that it requires less time to achieve high processing gain due to its coherent nature. The main drawback of this method is that a CR needs a dedicated receiver for every PU class. In cyclostationary feature detection technique, modulated signals are coupled with different pulses such as sine wave carriers, pulse trains, cyclic prefixes etc. resulting in built in periodicity [8]. Different cooperative sensing techniques can be applied in practical scenarios which may help the satellite users which are in deep fade region and reduce the HNP caused by failure in detecting the presence of PU due to presence of blocking obstacles and fading.

In DB technique, secondary system queries database to find out the unoccupied frequency bands and transmit in these bands. Therefore, there is no chance of co-channel interference between primary and secondary system. This technique has been proposed by FCC and OFCOM for using TVWS in secondary basis. The main drawback with this technique is that it mainly considers the static allocation of free bands and dynamic channel allocation seems to be difficult to implement in this scenario.

Regarding overlay techniques, encoding techniques further depend up on the level of interference between PU and cognitive user. When both cognitive and primary interference is strong, superposition coding can be used at the transmitters, while interference is decoded and removed at the receiver-side [44]. When both cognitive and primary interference is weak, rate splitting produces two streams from each transmitter, so that the first interfering streams can be decoded and removed while the second has to be tolerated as interference [45]. In case of weak primary link and strong cognitive interference, rate splitting technique can be combined with relaying for improved performance. Finally, in case of strong primary link and weak cognitive interference, known interference precancelation [31] can be applied. It should be noted that time sharing among above different techniques can be employed so that communication strategy adapts to the channel conditions. Therefore, different encoding techniques can be applied based on the signal interference level i.e. based on geographical location.

Regarding Underlay technique, different interference control and resource allocation techniques can be applied. The interference constraint in underlay scenario can be met by applying following methods:

- Multiple antennas: Suitable beamforming techniques can be applied to reduce interference to PUs.

- Wide Bandwidth: Cognitive signal is spread below noise floor and de-spread at the cognitive receiver.

- Power control: Suitable power levels are applied at the transmitter to ensure that the signal is below threshold.

Different transmit beamforming and IA techniques can be applied to mitigate the interference to PU. Underlay techniques

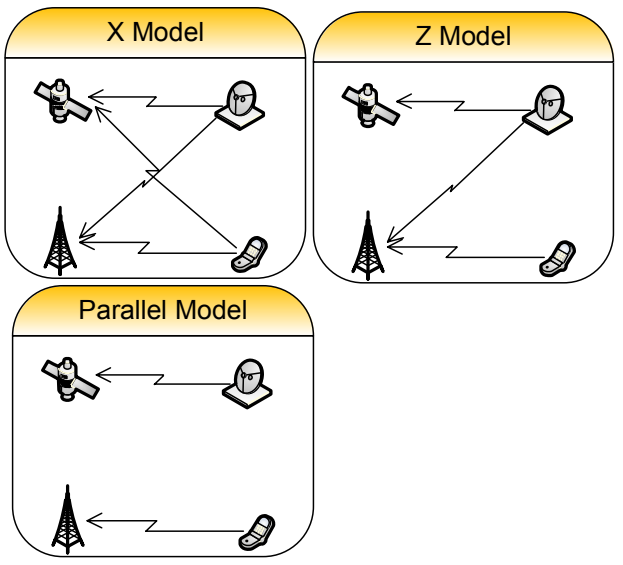

Fig. 4: Different co-existence models

can be applied in satellite cognitive scenario by exploring additional degrees of freedom such as polarization, radiation pattern of antenna, elevation angle etc. Scheduling in combination with beamforming can be an efficient method to implement in the context of considered coexistence scenario. Scheduling technique can be used to pick the users which are not getting in the beam's way under the constraint that it does not cause any harm to the primary user.

\section{CONClusion}

In this paper, firstly, we discussed different cognitive techniques referring to the current state of art in the context of satellite communication. Based on results obtained from the interference modeling between terrestrial BS and SAT terminal, it has been observed that SAT terminals near polar region get more interference from a terrestrial BS than the SAT terminals located near the equator due to variation in elevation angle. Furthermore, it can be noted that interference on SAT terminal from terrestrial BS becomes worse if we move from $52^{\circ}$ to the North. Moreover, it can be concluded that different cognitive techniques can be considered based on interference power level. SS and DB techniques seem to provide best performance in high interference region and in low or medium interference region, interference from secondary system to primary system can be suppressed by using some form of underlay technique. Although overlay technique can be used in both high interference and low interference scenario by using advanced coding and suitable modulation, it appears to be suitable only for integrated systems with very high level of interaction. Interference modeling using a unified framework by considering aggregate secondary network interference and it's impact on primary network in the context of cognitive SatComs will be our future work in this area.

\section{ACKNOWLEDGEMENT}

This work was supported by the National Research Fund, Luxembourg under AFR (Aids Training-Research) grant for $\mathrm{PhD}$ project on "Spectrum Sensing, Resource Allocation and 
Resource Management Strategies for Satellite Cognitive Communications", under the CORE project "CO2SAT: Cooperative and Cognitive Architectures for Satellite Networks" and by COST Action IC0902: "Cognitive Radio and Networking for Cooperative Coexistence of Heterogeneous Wireless Networks".

\section{REFERENCES}

[1] FCC, "Spectrum policy task force report," ET Docket 02-155, Nov. 02 2002.

[2] P. Chini, G. Giambene, and S. Kota, "A survey on mobile satellite systems," Int. J. Satell. Commun. Network, vol. 28, no. 1, pp. 29-57, 2009.

[3] S. Kandeepan, L. De Nardis, M.-G. Di Benedetto, A. Guidotti, and G. Corazza, "Cognitive satellite terrestrial radios," in IEEE GLOBECOM, dec. 2010, pp. $1-6$.

[4] B. Evans, M. Werner, E. Lutz, M. Bousquet, G. Corazza, G. Maral, and R. Rumeau, "Integration of satellite and terrestrial systems in future multimedia communications," Wireless Comm., IEEE, vol. 12, no. 5, pp. $72-80$, oct. 2005 .

[5] L. Gavrilovska and V. Atanasovski, "Resource management in wireless heterogeneous networks (whns)," in Telecomm. in Modern Satellite, Cable, and Broadcasting Services, 9th Int. Conf. on, oct. 2009, pp. 97 $-106$.

[6] S. Kota, "Hybrid/integrated networking for ngn services," in Wireless VITAE, 2nd Int. Conf. on, March 2011, pp. 1 -6.

[7] J. Mitola and G. Maguire, "Cognitive radio: making software radios more personal," Personal Comm., IEEE, vol. 6, no. 4, pp. 13 -18, aug 1999.

[8] A. Goldsmith, S. Jafar, I. Maric, and S. Srinivasa, "Breaking spectrum gridlock with cognitive radios: An information theoretic perspective," Proceedings of the IEEE, vol. 97, no. 5, pp. $894-914$, may 2009.

[9] X. Hong, C.-X. Wang, and J. Thompson, "Interference modeling of cognitive radio networks," in Veh. Techn. Conf., 2008 IEEE, may 2008, pp. $1851-1855$.

[10] A. Rabbachin, T. Quek, H. Shin, and M. Win, "Cognitive network interference," Selected Areas in Comm., IEEE Journal on, vol. 29, no. 2, pp. $480-493$, february 2011.

[11] _ , "Cognitive network interference- modeling and applications," in Communications, IEEE International Conference on, june 2011, pp. 1 $-6$.

[12] K. Ruttik, K. Koufos, and R. Jantti, "Computation of aggregate interference from multiple secondary transmitters," Comm. Letters, IEEE, vol. 15, no. 4, pp. $437-439$, april 2011.

[13] J. A. D. Ahn, H. Kim and D. Park, "Integrated/hybrid satellite and terrestrial networks for satellite imt-advanced services," Int. J. Satell. Commun. Network, 2010.

[14] M. Haddad, M. Debbah, and A. Hayar, "Distributed power allocation for cognitive radio," in Signal Processing and Its Applications, 2007. ISSPA 2007. 9th International Symposium on, feb. 2007, pp. $1-4$.

[15] E. Del Re, G. Gorni, L. Ronga, and R. Suffritti, "A power allocation strategy using game theory in cognitive radio networks," in Game Theory for Networks, Int. Conf. on, may 2009, pp. $117-123$.

[16] E. Del Re, F. Argenti, L. Ronga, T. Bianchi, and R. Suffritti, "Power allocation strategy for cognitive radio terminals," in Cognitive Radio and Advanced Spectrum Management, First Int. Workshop on, feb. 2008, pp. $1-5$

[17] X. Lin and Y. Fang, "A survey on the available digital tv bands for cognitive radio operations," in Comm. and Net. in China, Fourth Int. Conf. on, aug. 2009, pp. $1-7$.

[18] Ofcom, "Statement on cognitive access to interleaved spectrum," 2009, July 2009.

[19] J. Bradford, T. Cook, D. Ramsbottom, and S. Jones, "Optimising usage of spectrum below $15 \mathrm{ghz}$ used for defence in the uk," in Cognitive Radio and Software Defined Radios: Technologies and Techniques, 2008 IET Seminar on, sept. 2008, pp. $1-5$.

[20] S. Srinivasa and S. Jafar, "The throughput potential of cognitive radio: A theoretical perspective," in Signals, Systems and Computers, Fortieth Asilomar Conf. on, nov. 2006, pp. $221-225$.

[21] T. Clancy and B. Walker, "Spectrum shaping for interference management in cognitive radio networks," in SDR Forum Tech. Conf., 2006.
[22] D. Cabric, S. Mishra, and R. Brodersen, "Implementation issues in spectrum sensing for cognitive radios," in Signals, Systems and Computers, Conf. Record of the Thirty-Eighth Asilomar Conf. on, vol. 1, nov. 2004, pp. $772-776$.

[23] S. K. Sharma, S. Chatzinotas, and B. Ottersten, "Exploiting polarization for spectrum sensing in cognitive satcoms," in 7th Int. Conf. on Cognitive Radio Oriented Wireless Networks, June 2012.

[24] — - "Spectrum sensing in dual polarized fading channels for cognitive satcoms," in Globecom Conf., IEEE, Dec. 2012, accepted.

[25] A. Ghasemi and E. S. Sousa, "Fundamental limits of spectrum-sharing in fading environments," Wireless Comm., IEEE Trans. on, vol. 6, no. 2, pp. $649-658$, feb. 2007

[26] FCC, "Establishment of an interference temperature metric to quantify and manage interference and to expand available unlicensed operation in certain fixed, mobile and satellite frequency bands," ET Docket 0.-289, 2003.

[27] T. W. Ban, W. Choi, B. C. Jung, and D. K. Sung, "Multi-user diversity in a spectrum sharing system," Wireless Communications, IEEE Trans. on, vol. 8 , no. 1 , pp. $102-106$, jan. 2009.

[28] V. Cadambe and S. Jafar, "Interference alignment and degrees of freedom of the k user interference channel," Inf. Theory, IEEE Trans. on, vol. 54, no. 8, pp. $3425-3441$, aug. 2008.

[29] G. Zheng, S. Ma, K. kit Wong, and T.-S. Ng, "Robust beamforming in cognitive radio," Wireless Comm., IEEE Trans. on, vol. 9, no. 2, pp. 570 -576 , february 2010 .

[30] G. Zheng, K.-K. Wong, and B. Ottersten, "Robust cognitive beamforming with bounded channel uncertainties," Signal Processing, IEEE Trans. on, vol. 57, no. 12, pp. $4871-4881$, dec. 2009.

[31] M. Costa, "Writing on dirty paper (corresp.)," Inf. Theory, IEEE Trans. on, vol. 29 , no. 3 , pp. $439-441$, may 1983 .

[32] S. I. Gel'fand and M. S. Pinsker, "Probl. contr. and inform. theory," Coding for channel with random parameters, vol. 9, no. 1, pp. 19-31, 1980.

[33] Y. H. Yun and J. H. Cho, "An orthogonal cognitive radio for a satellite communication link," in Personal, Indoor and Mobile Radio Communications, IEEE 20th Int. Symp. on, sept. 2009, pp. 3154 -3158.

[34] L. Li, F. Khan, M. Pesavento, and T. Ratnarajah, "Power allocation and beamforming in overlay cognitive radio systems," in Veh. Tech. Conf., IEEE $73 \mathrm{rd}$, may 2011 , pp. 1 -5.

[35] J. Riihijarvi, P. Mahonen, M. Petrova, and V. Kolar, "Enhancing cognitive radios with spatial statistics: From radio environment maps to topology engine," in Cognitive Radio Oriented Wireless Networks and Comm., 4th Int. Conf. on, june 2009, pp. 1 -6.

[36] Y. Zhao, L. Morales, J. Gaeddert, K. Bae, J.-S. Um, and J. Reed, "Applying radio environment maps to cognitive wireless regional area networks," in New Frontiers in Dynamic Spectrum Access Networks, 2nd IEEE Int. Symposium on, april 2007, pp. $115-118$.

[37] J. wu Zhang, Q. Zhao, and J. yuan Zou, "The ieee802.22 wran system based on radio environment map (rem)," in Education Technology and Computer Science, First Int. Workshop on, vol. 1, march 2009, pp. 98 -101 .

[38] S. Kawade and M. Nekovee, "Wireless options for high data-rate indoor users: Cognitive access to tv white space," in First UK-India Int. Workshop on Cognitive Wireless Systems, April 2011.

[39] S. Chatzinotas, G. Zheng, and B. Ottersten, "Joint precoding with flexible power constraints in multibeam satellite systems," in GLOBECOM, IEEE, dec. 2011, pp. $1-5$

[40] M. Diaz, N. Courville, C. Mosquera, G. Liva, and G. Corazza, "Nonlinear interference mitigation for broadband multimedia satellite systems," in Satellite and Space Comm., Int. Workshop on, sept. 2007, pp. $61-65$.

[41] W. Perkins, "Fcc interference temperature gives licensees a fever," online, http://www.avw.co.nz/catalogue/images/andrew/docs/TP100323-EN.pdf.

[42] F. Digham, M.-S. Alouini, and M. Simon, "On the energy detection of unknown signals over fading channels," in Comm., IEEE Int. Conf. on, vol. 5 , may 2003 , pp. $3575-3579$.

[43] H. Urkowitz, "Energy detection of unknown deterministic signals," Proceedings of the IEEE, vol. 55, no. 4, pp. 523 - 531, april 1967.

[44] M. Costa and A. Gamal, "The capacity region of the discrete memoryless interference channel with strong interference (corresp.)," Inf. Theory, IEEE Transactions on, vol. 33, no. 5, pp. 710 - 711, sep 1987.

[45] A. Carleial, "Interference channels," Inf. Theory, IEEE Trans. on, vol. 24, no. 1 , pp. 60-70, jan 1978. 